\title{
Factores socioculturales relacionados al cumplimiento de los esquemas de vacunación en menores de 2 años durante el confinamiento
}

Sociocultural factors related to compliance with vaccination schemes in children under 2 years of age during confinement

Fatores socioculturais relacionados ao cumprimento dos esquemas de vacinação em crianças menores de 2 anos durante o confinamento

\author{
Johanna Carolina Quirola Gavilánez \\ jquirola9208@uta.edu.ec \\ Universidad Técnica de Ambato - Ecuador \\ https://orcid.org/0000-0003-1020-8019
}

\author{
José Luís Herrera López \\ jl.herrera@uta.edu.ec \\ Universidad Técnica de Ambato - Ecuador \\ http://orcid.org/0000-0002-2196-4875
}

\begin{abstract}
RESUMEN
Introducción: El incumplimiento de los esquemas de vacunación afecta significativamente la salud y el desarrollo sano y normal de niños y niñas. Existen varios factores, en especial socioculturales que pueden influir en el incumplimiento de los esquemas de vacunación. Esto a su vez se ha visto agravado más debido a la presencia de la pandemia del Covid19. Objetivo: Identificar los factores socioculturales relacionados al cumplimiento del esquema de vacunación en menores de 2 años en una muestra de madres y padres del Ecuador. Metodología: La metodología empleada para el análisis del estudio comprende un diseño descriptivo, cuantitativo, comparativo, de corte transversal, el tiempo del estudio comprende entre marzo de 2020 y agosto de 2021. Participantes: Padres de familia de 208 niños menores de 2 años con esquemas atrasados que son atendidos en el Centro de Salud $\mathrm{N}^{\circ} 1$ de la ciudad de Ambato. Resultados: Los principales factores asociados con el incumplimiento de los esquemas de vacunación comprende la situación socioeconómica de los padres, la edad de los padres, la movilidad y el transporte y el acceso a las vacunas. También la presencia del covid-19 y las medidas sociosanitarias han incidido en la dificultad de adherencia a los esquemas de vacunación. Conclusiones: Las limitantes actuales en los esquemas de adherencia a la vacunación inciden fuertemente en el cumplimiento de los programas de vacunación y deben ser tomados en cuenta para los respectivos correctivos en las políticas públicas sanitarias.
\end{abstract}

Palabras clave / Descriptores: Confinamiento, Covid-19, factor, niños, vacunación

\begin{abstract}
Introduction: Failure to comply with vaccination schedules significantly affects the health and healthy and normal development of boys and girls. There are several factors, especially sociocultural, that may influence non-compliance with vaccination schedules. This in turn has been further aggravated due to the presence of the Covid-19 pandemic. Objective: To identify the socio-cultural factors related to compliance with the vaccination scheme in children under 2 years of age in a sample of mothers and fathers from Ecuador. Methodology: The methodology used for the analysis of the study comprises a descriptive, quantitative, comparative, cross-sectional design, the study time is between March 2020 and August 2021. Participants: Parents of 208 children under 2 years of age with Overdue schemes that are treated at the Health Center No. 1 in the city of Ambato. Results: The main factors associated with non-compliance with vaccination schedules include the socio-economic situation of the parents, the age of the parents, mobility and transportation, and access to vaccines. Also, the presence of covid-19 and social health measures have affected the difficulty of adherence to vaccination schedules. Conclusions: The current limitations in the vaccination adherence schemes strongly affect compliance with the vaccination programs and must be taken into account for the respective corrections in the public health policies.
\end{abstract}

Keywords: Confinement, Covid-19, factor, children, vaccination.

\section{RESUMO}

Introdução: $\mathrm{O}$ não cumprimento dos calendários de vacinação afeta significativamente a saúde e o desenvolvimento saudável e normal de meninos e meninas. Vários são os fatores, principalmente os socioculturais, que podem influenciar 
o não cumprimento dos calendários vacinais. Isso, por sua vez, se agravou ainda mais devido à presença da pandemia de Covid-19. Objetivo: Identificar os fatores socioculturais relacionados ao cumprimento do calendário vacinal em crianças menores de 2 anos em uma amostra de mães e pais do Equador. Metodologia: A metodologia utilizada para a análise do estudo inclui um desenho descritivo, quantitativo, comparativo, transversal, o tempo do estudo é entre março de 2020 e agosto de 2021. Participantes: Pais de 208 crianças menores de 2 anos com atraso regimes que são tratados no Centro de Saúde $n^{\circ} 1$ da cidade de Ambato. Resultados: Os principais fatores associados ao não cumprimento do calendário vacinal incluem a situação socioeconômica dos pais, idade dos pais, mobilidade, transporte e acesso às vacinas. A presença da covid-19 e as medidas socio-sanitárias também afetaram a dificuldade de adesão aos calendários de vacinação. Conclusões: As atuais limitações nos esquemas de adesão à vacinação têm forte impacto no cumprimento dos programas de vacinação e devem ser levadas em consideração para as respectivas correções nas políticas públicas de saúde.

Palavras-chave: Confinamento, Covid-19, fator, crianças, vacinação

\section{INTRODUCCIÓN}

Debido a las consecuencias emanadas del Covid-19 y las posteriores medidas de restricción sanitarias tomadas por los distintos gobiernos a fin de prevenir el contagio masivo. Se presentan dificultades y limitaciones en el desarrollo normal de los programas de vacunación en niños y niñas menores de 2 años en el Ecuador. Dentro de este contexto, se identifican en el país y el continente limitaciones recurrentes que afectan al cumplimiento adecuado de los calendarios de vacunación en los niños y niñas. Dichas limitaciones muchas veces son corregidas a través de campañas de vacunación y de la adecuada información y educación de los padres de familia que son responsables del cumplimiento de estos procesos. Y también limitaciones debido a las condiciones del Covid-19, que se suma a las problemáticas frecuentes de acceso a la vacunación. En este contexto, el análisis de los factores de riesgo que limitan el cumplimiento de la calendarización durante la pandemia actualmente se encuentra escasamente analizados y se desconoce la dinámica actual de funcionamiento, por lo que las medidas de estimulación de la vacunación por parte del Ministerio de Salud Pública del Ecuador pueden verse afectado por una falta de actualización de datos y de entendimiento de la problemática social actual. En base a estos antecedentes, el objetivo de la investigación es identificar los factores socioculturales relacionados al cumplimiento del esquema de vacunación en menores de 2 años en una muestra de madres y padres del Ecuador durante la pandemia actual.

A partir de esto, se realiza una revisión teórica acerca de los efectos de la vacunación en menores de 2 años y la eficacia de las mismas en la prevención de la mortandad y morbilidad. También se analizan los principales factores sociodemográficos que limitan el cumplimiento de la calendarización de vacunación, así como aquellos que se encuentran apareciendo a partir de la presencia del Covid-19 y las medidas sanitarias empleadas en los distintos países y el Ecuador. Posterior a ella se presenta un estudio de campo en padres y madres de familia de niños y niñas menores de 2 años a través de una metodología descriptiva de corte transversal para conocer sobre los factores socioculturales asociados a la vacunación. En la sección de resultados se muestran los principales datos de la evaluación a los padres de familia que se muestran a través de tablas de contingencia. Finalmente, en la sección de discusión se contrastan los resultados del estudio con la revisión preliminar en la fundamentación teórica y la revisión del estado del arte.

\section{FUNDAMENTO TEÓRICO}

Una visión actual de la vacunación es verla como un factor de desarrollo social, económico y de reducción de la pobreza (Buera et al., 2021; Porras, 2008). La vacunación de niños menores de cinco años es una de las medidas más eficaces que existen para reducir la mortalidad infantil (Moyer, 2019). 
El incumplimiento de los esquemas de vacunación afecta la salud y al desarrollo de niños y niñas (Díaz et al., 2018). Se estima que anualmente se evitan 2,5 millones de muertes debido al cumplimiento del calendario vacunación. Sin embargo, al menos el 20\% de los niños que nacen, no reciben estos beneficios y corren el riesgo de morir antes de los cinco años (Escobar-Díaz y otros, 2018) (Mendoza-Mendoza et al., 2020).

En los últimos años, los programas de vacunación en América Latina y en el Ecuador, se han incrementado de manera sostenida (Parcon-Bitanga et al., 2019). Esto debido a las políticas sanitarias y al desarrollo de la investigación enfocada en mejorar la efectividad de las vacunas. Sin embargo, este proceso se ha visto mermado, debido a la interrupción de los servicios de salud esenciales como resultado de la pandemia del SARS-CoV-2. Que afectó con mayor intensidad a los programas de vacunas de rutina y externas. A raíz de esto, la vacunación programática en 2020 disminuyó respecto al promedio 2015-2019 (Bastías et al., 2021; Carrasco-Fernandez et al., 2021).

\subsection{Factores de riesgo asociados a la vacunación de niños y niñas}

La vacunación es una parte integral del derecho a la salud (Martin-Fumadó et al., 2021) que tiene como objetivo ayudar a reducir la mortalidad infantil. Sin embargo, existen varios limitantes que afectan a este proceso. Principalmente los factores socioculturales.

En este aspecto, el género es uno de ellos. Por ejemplo, en tres de cada cuatro hogares, las mujeres cuidan exclusivamente a sus hijos; y la carga de cuidar es aún mayor en los grupos de menores ingresos económicos. Lo que aleja la posibilidad de apoyo compartido entre los progenitores (más aún en madres jóvenes, menores de 19 años) (Trinidad \& Gutiérrez, 2021).

También inciden la escolaridad básica, el desinterés y limitada adherencia a la vacunación (Sangoluisa-Rosales et al., 2019); la pertenencia a una minoría étnica, la ruralidad del domicilio, la religiosidad (Tirado Otálvaro \& Moreno Uribe, 2007), los bajos ingresos económicos, la responsabilidad laboral al menos uno de los padres, que los tiene fuera de casa, la movilidad y la transportación (Chavez Amaro et al., 2021; Isidro-Ríos \& Gutiérrez-Aguado; 2021; Villalobos Vásquez, 2019) e incluso la disponibilidad y el acceso a vacunas.

Junto a esto se añaden, las restricciones derivadas del Covid-19, que dificultan el complimiento del esquema de vacunación (Coello, 2021). Esto pese a los esfuerzos del Ecuador para cumplir con los programas de vacunación (Ministerio de Salud Pública, 2019; Ministerio de Salud de Argentina, 2020). Todo esto, lo convierte en un factor de riesgo potencial, aún mayor sumando a los factores antes señalados (de las Casas, 2021; Faundes et al., 2021; Oyola \& Murillo, 2020).

El riesgo se incrementa aún más, por la falta de datos epidemiológicos actualizados que permitan tomas de decisiones oportunas (Carrizo et al., 2017), preventivas y de lucha para la erradicación de enfermedades tratables a través de la vacunación. Por ejemplo, se evidencia que en los programas de vacunación durante el período crítico de la pandemia (2020 y 2021) existe una caída en la cobertura del $94,2 \%$ para la tuberculosis (BCG), del $88,8 \%$ para la difteria, tétanos y tosferina (DTP), del $91,1 \%$ para sarampión y del $88,9 \%$ en poliomielitis para niños de 19 a 35 meses (Bárcena, 2020). Además, la pandemia afectó la producción de las vacunas a nivel mundial, así como la oportuna distribución. Algo que se debe agregar también, es que la administración gubernamental anterior (2017-2021) en el Ecuador, no trató los biológicos de manera oportuna, dejando el programa de vacunación interrumpido. Ocasionando consecuencias desagradables futuras.

Esto se intentó aplacar con el actual gobierno que priorizó una inversión de 17 millones para obtener las dosis que necesita la población pediátrica (El Universo, 2021). Así como la negociación de donaciones con gobiernos amigos. Con ello, además, se creó la campaña nacional "Todo 
campeón está vacunado", que el Ministerio de Salud Pública (MSP) introdujo como una estrategia de vacunación estándar (no COVID-19) para los niños que no han sido vacunados a nivel nacional debido a retrasos en el calendario de vacunación. Para esto, también se acortó el intervalo de dosificación para asegurar la eficacia de la vacuna en los nacidos antes del 1 de agosto 2021 que no hayan recibido dosis completas en el año anterior (Ministerio de Salud, 2021; Pardo et al., 2007). A pesar de estos aspectos, aún no se tiene claro el efecto en el proceso de adherencia a las campañas de vacunación.

\section{PROCEDIMIENTOS METODOLOGICOS}

La metodología empleada para el análisis del estudio comprende un diseño descriptivo, cuantitativo, de corte transversal (Hernández \& Mendoza, 2018; Ato et al., 2013). El tiempo aplicado corresponde entre los meses de marzo de 2020 a agosto de 2021. Se pretende establecer los factores socioculturales relacionados al cumplimiento del esquema de vacunación en menores de 2 años durante el confinamiento, lo cual permitirá captar y realizar el seguimiento pertinente de los menores con vacunas pendientes.

\subsection{Población y muestra}

La población para el estudio, está estimada en 208 niños menores de 2 años con esquemas atrasados que son atendidos en el Centro de Salud $\mathrm{N}^{\circ} 1$ de la ciudad de Ambato. La muestra estimada mediante una ecuación (5\% de margen de error, 95\% de confiabilidad y 50\% de probabilidad de ocurrencia y no ocurrencia), establece que el tamaño corresponde a 135 niños (Solís et al., 2018).

\subsection{Procedimiento}

El presente reporte forma parte del proyecto de investigación "Factores socioculturales relacionados con el cumplimiento de los esquemas de vacunación en niños menores de 2 años durante el confinamiento" mismo que fue evaluado y aprobado por el Consejo de Escuela de la Facultad de Ciencias de la Salud de la Universidad Técnica de Ambato. Para el desarrollo del levamiento de dato se solicitó permiso de las autoridades del centro de salud que forma parte del estudio. Posteriormente, se informó a los padres de los menores acerca del propósito del estudio actual y se solicitó la firma del consentimiento informado para asegurar la participación voluntaria. Los sujetos de estudio fueron 135 padres de niños menores de 2 años atendidos en el centro de Salud $\mathrm{N}^{\circ} 1$ a cuyos padres se les aplicó la encuesta por medio de la captación y seguimiento oportuno del menor, tanto en el centro de salud y mediante visitas domiciliarias. Cabe señalar que los procesos investigativos empleados se ajustaron a los lineamientos éticos de la investigación con seres humanos de la Convención de Helsinki.

\subsection{Instrumento}

La recolección de datos se realizó mediante la aplicación de una encuesta sobre esquemas de vacunación en menores de un año (Sacba, 2020). Previamente se validó en la versión original mediante 6 madres de familia, analizando ajustes de diseño para su mejor comprensión. Posteriormente presentado y validado mediante el asesor de tesis para cumplir con criterios de 
métrica en ciencias sociales y de la salud (Moreta-Herrera et al., 2021). En el caso del presente estudio, la validación se realizó por medio de comité de expertos, quienes validaron la funcionalidad del mismo en el contexto ecuatoriano.

El instrumento en su base principal consta de dos secciones. En la primera sección se recolectan los datos informativos de las madres, mediante 7 preguntas cerradas. La segunda sección consta de 11 preguntas cerradas las cuales recolecta información acerca de los factores que influyen en la no adherencia del esquema de vacunación.

Para adaptar al estudio, se reestructuró la encuesta y se adicionó 5 preguntas que determinan la relación del confinamiento con el cumplimiento de la vacunación. El instrumento consta de un total de 24 preguntas.

\section{RESULTADOS Y DISCUSIÓN}

\subsection{Análisis de los factores socioeconómicos}

La tabla 1 presente el análisis de los factores socioeconómicos. Ahí se encontró, que de los padres encuestados el $48 \%$ tiene como ocupación un trabajo fuera de casa y el 13\% negocio propio y el 39\% se desempeña como ama de casa. Esto significa que, debido a las ocupaciones laborales, al menos una persona permanece fuera de casa durante varias horas del día lo que puede tener impacto en la atención y cuidado de menores en el hogar.

En el caso de accesibilidad, el 50\% utiliza el bus como medio de transporte. Esto revela que la mayoría acude a los centros de vacunación utilizando el sistema de servicio público, debido a su bajo costo, constante fluidez y porque se puede acceder a los puntos importantes de la ciudad. Ahondando en este aspecto, para el 19\% de los encuestados, los costos que derivan en transporte para acceso a asistencia en salud pública ven en ocasiones un factor limitante para acceder a la búsqueda de asistencia.

Tabla 1.

Factores socioeconómicos

\begin{tabular}{llrrr}
\hline \multicolumn{2}{l}{ Factores Socioeconómicos } & Frecuencia & Porcentaje \\
\hline \multirow{2}{*}{ Ocupación } & Ama de casa & 53 & $39 \%$ \\
& Negocio propio & 17 & $13 \%$ \\
& Trabaja fuera de casa & 65 & $48 \%$ \\
\hline \multirow{2}{*}{ Accesibilidad } & \multicolumn{2}{c}{ Total } & $\mathbf{1 3 5}$ & $\mathbf{1 0 0 \%}$ \\
& Vehículo propio & 27 & $20 \%$ \\
& Bus & 67 & $50 \%$ \\
& Caminando & 41 & $30 \%$ \\
\hline \multirow{2}{*}{ Gastos de transporte como } & Total & $\mathbf{1 3 5}$ & $\mathbf{1 0 0 \%}$ \\
obstáculo & Sí & & 0 & $0 \%$ \\
& No & & 109 & $81 \%$ \\
& A veces & & 26 & $19 \%$ \\
\hline
\end{tabular}

Fuente: Encuesta

Elaborado por: Quirola (2021)

\subsection{Análisis de los factores culturales}


En la tabla 2 se observa que el $81 \%$ de los participantes se autoidentifica como mestizo(a) y el resto de la distribución, se reparten entre indígenas o afro-ecuatorianos. Además, mayoritariamente se identifican como católicos $(61 \%)$. En general, los participantes comulgan con el cristianismo. Acerca, de la importancia que tienen las vacunas, en la totalidad de los encuestados, se identificó que, para ellos, la vacunación es importante. Esto que significa que quienes integran el estudio están al pendiente de que sus hijos reciban las vacunas que les corresponde; más aún al tratarse de los menores de edad para que su sistema inmunológico se vea reforzado.

Tabla 2.

Factores culturales

\begin{tabular}{llrrr}
\hline & Factores Culturales & Frecuencia & Porcentaje \\
\hline \multirow{3}{*}{ Etnia } & Mestizo & 109 & $81 \%$ \\
& Indígena & 24 & $18 \%$ \\
& Afro ecuatoriano & 2 & $1 \%$ \\
\hline \multirow{3}{*}{ Religión } & Total & $\mathbf{1 3 5}$ & $\mathbf{1 0 0 \%}$ \\
& Católica & & 82 & $61 \%$ \\
& Evangélica & & 53 & $39 \%$ \\
& Otro & & 0 & $0 \%$ \\
\hline Considera las vacunas & & Total & $\mathbf{1 3 5}$ & $\mathbf{1 0 0 \%}$ \\
importantes & Sí & & 135 & $100 \%$ \\
\hline & No & & 0 & $0 \%$ \\
\hline
\end{tabular}

Fuente: Encuesta

Elaborado por: Quirola (2021)

\subsection{Análisis de los Factores sociodemográficos}

En la tabla 3, se evidencia que el $49 \%$ de los participantes, tienen hijos e hijas con edades entre los 7 a 9 meses, seguidos por aquellos que tienen de 1 a 2 años. Además, el 66\% de las madres tienen entre 20 a 30 años; de lo que se interpreta que el grupo etario que cubre la tenencia de hijos corresponde a los jóvenes.

Acerca del número de hijos, en su mayoría (83\%) los participantes del estudio, tienen entre 1 a 2 hijos o hijas. Estos valores, son un indicativo de que las parejas procuran tener pocos hijos en su mayoría. Es probable que debido a las condiciones económicas y sociales en la actualidad resultan más difíciles en aspectos la manutención de más hijos de los mencionados.

Sobre el estado civil, el $44 \%$ están bajo la modalidad de unión libre, seguido por los casados y casadas, aunque en menor proporción (35\%).

En cuanto a la escolaridad, el 53\% tiene una formación secundaria con un título de bachillerato, seguido por aquellos con formación superior universitaria en menor proporción y por aquellos únicamente con estudios primarios.

Respecto a la sectorización del domicilio, el 47\% provienen de la parroquia La Merced y del centro urbano de Ambato, lo cual resulta ser cercano a la ubicación en la que se encuentra el Centro de Salud del presente estudio. 
Tabla 3.

Factores sociodemográficos

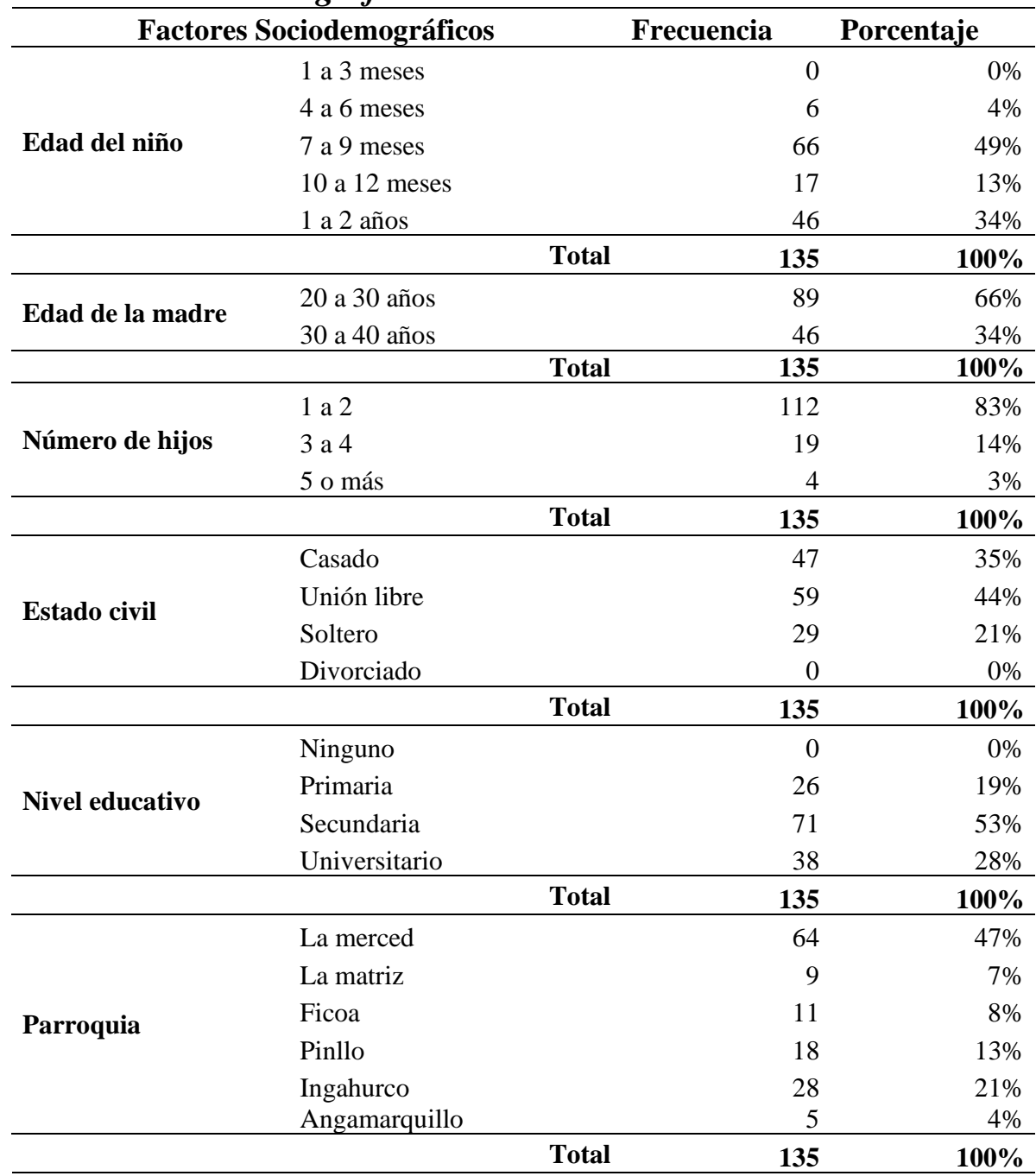

Fuente: Encuesta

Elaborado por: Quirola (2021)

\subsection{Análisis de la dinámica del cumplimiento de la vacunación}

En la tabla 4, se observa que el $95 \%$ de los padres tuvo que esperar por la vacuna de sus hijos menos de 30 minutos. Dado el corto tiempo, se observa en la mayoría de los casos que el proceso de coordinación de la vacuna es eficiente. Además, que generalmente, reciben en los centros de atención un trato cortés y educado por parte de los profesionales encargados. Por otra parte, un $24 \%$ identifica dificultades en el acceso a la vacuna que les corresponde, debido a que no todos los centros cuentan con la cobertura requerida.

También, se observa, que la mayoría de los padres reciben por parte de los encargados información sobre la vacuna que será administrada, que incluye conocimiento sobre las enfermedades que previene, las contraindicaciones y las precauciones que deben tomar al experimentar algún tipo de reacción. En este aspecto, el $88 \%$ dice que conoce de las posibles reacciones luego de la vacunación; sin embargo, un 14\% lo desconoce. Una proporción representativa (34\%) no conoce de las vacunas que debe recibir el menor de un año. Esto es relevante porque puede incidir en el incumplimiento de la programación de vacunas que debe recibir el niño.

Acerca del cumplimiento del agendamiento de la vacunación, el 29\% apenas cumple con las fechas señaladas que le corresponde a la inmunización del niño o niña. En el resto de condiciones se 
cumple parcialmente o no lo cumplen por olvido. Esto es relevante, dado que tienen un entendimiento claro de su importancia. En esta línea de pensamiento, el $79 \%$ considera que sí presentó dificultades para asistir a la vacunación, principalmente por razones asociadas al trabajo debido a que requerían permiso laboral.

Respecto a los factores asociados a la pandemia del covid-19, el $87 \%$ considera que la pandemia sí influyó en el atraso de la vacunación. Además, el 90\% manifiesta que el confinamiento sí le generó limitación para asistir a la vacunación (debido a la cuarentena y la desconfianza de salir y contagiarse). Finalmente, el 95\% considera que en el confinamiento se ajustaron los horarios de atención y que se tuvieron que modificar el proceso de vacunación, aun así, existió apertura del centro de salud los días de asistencia pese a las reprogramaciones dadas.

Tabla 4.

Cumplimiento de la vacuna

\begin{tabular}{|c|c|c|c|}
\hline \multicolumn{2}{|c|}{ Cumplimiento de la vacuna } & Frecuencia & \multirow{2}{*}{$\frac{\text { Porcentaje }}{95 \%}$} \\
\hline Tiempo de espera para la & Menos de 30 minutos & 128 & \\
\hline vacuna & 31 minutos a 1 hora & 7 & $5 \%$ \\
\hline & Total & 135 & $100 \%$ \\
\hline \multirow{4}{*}{ Trato recibido es bueno } & Sí & 126 & $93 \%$ \\
\hline & No & 0 & $0 \%$ \\
\hline & A veces & 9 & $7 \%$ \\
\hline & Total & 135 & $100 \%$ \\
\hline \multirow{3}{*}{$\begin{array}{l}\text { Encuentra vacunas } \\
\text { disponibles }\end{array}$} & $\overline{\text { Sí }}$ & 103 & $76 \%$ \\
\hline & No & 32 & $24 \%$ \\
\hline & Total & 135 & $100 \%$ \\
\hline \multirow{3}{*}{$\begin{array}{l}\text { Ha recibido información } \\
\text { sobre vacunas }\end{array}$} & Sí & 129 & $96 \%$ \\
\hline & No & 6 & $4 \%$ \\
\hline & Total & 135 & $100 \%$ \\
\hline \multirow{4}{*}{$\begin{array}{l}\text { Conoce las reacciones luego } \\
\text { de la vacunación }\end{array}$} & Sí & 119 & $88 \%$ \\
\hline & No & 0 & $0 \%$ \\
\hline & Desconoce & 16 & $12 \%$ \\
\hline & Total & 135 & $100 \%$ \\
\hline \multirow{3}{*}{$\begin{array}{l}\text { Conoce las vacunas que } \\
\text { debe recibir su niño menor } \\
\text { de un año }\end{array}$} & Sí & 89 & $66 \%$ \\
\hline & No & 46 & $34 \%$ \\
\hline & Total & 135 & $100 \%$ \\
\hline \multirow{4}{*}{$\begin{array}{l}\text { Ha cumplido con las citas } \\
\text { de vacunación }\end{array}$} & Siempre & 39 & $29 \%$ \\
\hline & A veces & 67 & $50 \%$ \\
\hline & Se me olvida & 29 & $21 \%$ \\
\hline & Total & 135 & $100 \%$ \\
\hline \multirow{3}{*}{$\begin{array}{l}\text { Existieron dificultades para } \\
\text { asistir a la vacuna }\end{array}$} & Sí & 106 & $79 \%$ \\
\hline & No & 29 & $21 \%$ \\
\hline & Total & 135 & $100 \%$ \\
\hline \multirow{3}{*}{$\begin{array}{l}\text { Considera que la pandemia } \\
\text { influyó en el atraso de la } \\
\text { vacuna }\end{array}$} & Sí & 118 & $87 \%$ \\
\hline & No & 17 & $13 \%$ \\
\hline & Total & 135 & $100 \%$ \\
\hline \multirow{3}{*}{$\begin{array}{l}\text { El confinamiento le generó } \\
\text { limitación para asistir a la } \\
\text { vacuna }\end{array}$} & Sí & 121 & $90 \%$ \\
\hline & No & 14 & $10 \%$ \\
\hline & Total & 135 & $100 \%$ \\
\hline \multirow{3}{*}{$\begin{array}{l}\text { Durante el confinamiento el } \\
\text { horario de atención se ajustó }\end{array}$} & Sí & 128 & $95 \%$ \\
\hline & No & 7 & $5 \%$ \\
\hline & Total & 135 & $100 \%$ \\
\hline \multirow{3}{*}{$\begin{array}{l}\text { Hubo apertura del centro de } \\
\text { salud los días que asistió }\end{array}$} & $\overline{\text { Sí }}$ & 128 & $95 \%$ \\
\hline & No & 7 & $5 \%$ \\
\hline & Total & 135 & $100 \%$ \\
\hline
\end{tabular}

Fuente: Encuesta

Elaborado por: Quirola (2021) 


\subsection{Discusión}

La inmunización ha demostrado ser una medida eficaz para reducir la morbilidad y mortalidad infantil a nivel mundial, capaz de evitar enfermedades infecciosas y de esta manera prevenir discapacidades, comorbilidades y muertes. Sin embargo, a razón de la pandemia del Covid-19, las coberturas de vacunación se han visto afectadas por las diferentes medidas sanitarias, económicas y sociales empleadas en el país (Ministerio de Salud Pública, 2019; Ministerio de Salud de Argentina, 2020).

Según los resultados encontrados, en la tabla 1 en los factores socioeconómicos el $48 \%$ de padres encuestados trabajan fuera de casa, un 50\% llega al centro de salud en bus, y el $19 \%$ manifiesta que los gastos de transporte son un obstáculo para acercarse a la vacuna agendada. La evidencia refleja que el factor socioeconómico, las actividades laborales y el acceso al transporte inciden de alguna manera en el adecuado cumplimiento de la vacunación infantil. Esto concuerda con estudios similares llevados en otros países como Perú por ejemplo (Isidro-Ríos \& GutiérrezAguado; 2021; Villalobos Vásquez, 2019) o Cuba (Chavez Amaro et al., 2021). En este aspecto, se requiere investigar este hecho dentro de los factores socioeconómicos de cada unidad de salud.

Por otra parte, en la tabla 2 en los factores culturales el $81 \%$ de los encuestados son mestizos este grupo también representa una mayoría a nivel nacional, el $61 \%$ tiene el catolicismo como religión y el $100 \%$ de los padres dicen que las vacunas son importantes, lo que significa que toda la población del estudio ha estado esperando que las vacunas se desarrollen con normalidad. Contrastando esta información con los resultados de una encuesta realizada en el Centro de Salud $\mathrm{N}^{\circ} 3$ de la provincia de Chimborazo de 74 personas encuestadas el $63.5 \%$ se identifica con etnia mestiza y el $100 \%$ considera que la etnia no influye en el cumplimiento del esquema de vacunación en sus niños. Por lo que se entiende que los datos tienen pertinencia dentro de la misma región (Benavides, 2019).

En lo que respecta a los factores sociodemográficos, el $49 \%$ manifiesta que la edad de los niños es de 7 a 9 meses. El 66\% de madres tienen edades entre los 20 a 30 años, lo cual evidencia que el grupo etario corresponde a la juventud y que concuerda con la tenencia sociodemográfica en el Ecuador. Además, el $83 \%$ tienen entre 1 a 2 hijos o hijas. Además, el $44 \%$ tienen como estado civil la unión libre. El 53\% tiene una formación secundaria, que corresponde a haber cursado el colegio. El $47 \%$ provienen de la parroquia La Merced, sector donde se ubica el Centro de Salud $\mathrm{N}^{\circ} 1$. Comparando los resultados con un estudio realizado en el establecimiento de salud Vergeles en la Cantón Milagro de 15 personas encuestadas el 60\% corresponde a la edad de 30 a 36 años, predominando el estado civil unión libre en $53,3 \%$, la residencia proveniente corresponde a la zona urbana en $73,3 \%$, la instrucción formativa el $40 \%$ ha culminado la secundaria, demostrando similitud en los resultados de ambos centros de salud determinando la relación del nivel académico con el cumplimiento de la vacuna agendada (Sarmiento, 2020).

Sobre la dinámica de la vacunación en el presente, el 95\% recibió atención en un tiempo menor a los 30 minutos, lo cual señala un proceso de vacunación eficaz, además de percibir un buen trato en la atención. Esto coincide con las actuales políticas de asistencia y trato en los programas de vacunación y los ODS (Mendoza-Mendoza et al., 2020; Parcon-Bitanga et al., 2019). Sin embargo, cabe señalar que una proporción representativa presentó dificultades en la disponibilidad de la vacuna, por lo que tuvieron que buscar en diferentes centros de salud de la ciudad y a nivel privado para poder acceder a las misma.

También se evidencia niveles adecuados de información sobre la vacuna, los efectos y las contradicciones por parte del personal de salud y además los padres están al tanto de dicha información. Estos aspectos concuerdan como se señaló con los procesos de involucramiento en los grupos de atención para la fomentación de la vacunación a través de la información (MartinFumadó et al., 2021). Aun así, se evidencia dificultades en el cumplimiento de los padres para ajustarse a los cronogramas de vacunación. Las limitaciones coinciden con los estudios 
referenciales previos que estiman al trabajo, la movilidad, la sectorización del domicilio y las dificultades laborales como factores de riesgo (Coello, 2021).

El 79\% piensa que sí hubo dificultades para asistir a la vacunación ya que les generó miedo de contagiarse de COVID-19. El 87\% dice que la pandemia sí influyó en el atraso de la vacunación infantil debido a las medidas de bioseguridad que se habían establecido. El 90\% opina que el confinamiento sí generó limitación para asistir a la vacuna. El 95\% opina que en el confinamiento el horario de atención sí se ajustó. El 95\% opina que hubo apertura del centro de salud los días que asistió. Según el estudio de Oyola y Murillo (Oyola \& Murillo, 2020) se reflejó que el 54\% dijo que el establecimiento de salud estuvo cerrado los primeros meses de pandemia, además el $48 \%$ respondió que no se acercó al centro de salud para la vacuna por miedo a contagiarse y un $28 \%$ no encontró la vacuna el día de su agendamiento. Al comparar los resultados se puede observar una diferencia en la apertura del establecimiento, lo cual puede ser explicado por la naturaleza epidemiológica que tuvo el Covid-19 en distintas ciudades del Ecuador. Por el contrario, se puede encontrar un porcentaje similar respecto a las consecuencias dadas tras la pandemia, algunas madres se atrasaron de la vacuna por miedo a contagiarse en el establecimiento de salud.

La presencia del Covid-19 en el contexto del Ecuador según la percepción de los participantes establece que ha incidido en el desarrollo normal del proceso de vacunación de los infantes, sobre todo en el cumplimiento del esquema de vacunación y a posterior en la adherencia de los padres a la misma (Ministerio de Salud Pública, 2019; Ministerio de Salud de Argentina, 2020). Esto está generando dificultad y mayor riesgo de problemas de salud a futuro debido al enorme limitante ocasionado por el Covid-19. Similar situación se observa a partir de las conclusiones de investigaciones en el continente (de las Casas, 2021; Faundes et al., 2021; Oyola \& Murillo, 2020), aunque en el caso del Ecuador no se presentan estudios similares

\section{CONCLUSIÓN}

Se concluye que los participantes se encuentran dentro de un marco socioeconómico de clase media, con criterios demográficos dentro del grupo etario de los participantes como jóvenes. Además, dada estas condiciones pueden verse implicadas algunas dificultades de adherencia al esquema de vacunación de los hijos e hijas.

Resalta que los participantes evaluados conocen de la importancia que tiene la vacunación en los hijos para el desarrollo sano y normal. Aunque cuentan con mucha información de la vacunación que incluye los beneficios y contraindicaciones de las mismas, sin embargo, eso no impide que presenten dificultades de adherencia a los esquemas de vacunación.

Los principales factores que limitan la adherencia corresponden a la situación socioeconómica, los niveles de escolaridad, las actividades laborales, la movilidad y la disponibilidad de vacunas. Cabe señalar que a estos elementos se le debe considerar la pandemia actual del Covid-19 que ha incidido más en estos limitantes ya comentados.

Por la tanto, para corregir estos limitantes se considera importante extender las campañas de vacunación, acercar la asistencia de este proceso a diversas zonas de la ciudad en especial de las más lejanas y también mejorar los parámetros de actuación considerando las particularidades específicas de la pandemia.

\section{REFERENCIAS}

Ato, M., López, J., \& Benavente, A. (2013). Un sistema de clasificación de los diseños de investigación en psicología. Anales de Psicología, 29(3), 1038-1059. https://doi.org/10.6018/analesps.29.3.178511 
Bárcena, A. (2020). Salud en las Américas. Resumen: panorama regional y perfiles de país. Recuperado el 19 de Octubre de 2021 de: https://www.paho.org/salud-en-las-americas-2017/wp-content/uploads/2017/09/PrintVersion-Spanish.pdf

Bastías, M., Brstilo, I., \& González, C. (2021). Vacunación programática 2020 en Chile en tiempos de pandemia por SARS-CoV-2. Revista chilena de infectología, 38(3), 11-30. Recuperado el 16 de Octubre de 2021, de https://www.scielo.cl/scielo.php?script=sci_arttext\&pid=S0716$10182021000300355 \& \operatorname{lng}=$ es\&nrm=iso\&tlng=es

Benavides, L. (2019). Factores que influyen en la no adherencia al esquema de vacunación de la población infantil en el centro de salud $n^{\circ} 3$ de la Provincia de Chimborazo (Trabajo de investigación no publicado ed.). Ambato: Universidad Técnica de Ambato. Recuperado el 30 de 10 de 2021, de Repositorio.uta.edu.ec: https://repositorio.uta.edu.ec/bitstream/123456789/30419/2/Proyecto\%20de\%20Investigacion\%20.pdf

Buera, M. M., Pérez, A. M., García, M. P., Fernández, T. M., García, V. P., \& Aranda, N. D. (2021). Estado actual y conflictos ético/morales de las vacunas en niños. Revista Sanitaria de Investigación, 2(4), 61.

Carrasco-Fernandez, J. A., Arriola-Aponte, M. E., \& Diaz-Manchay, R. (2021). Privación y reactivación del servicio de vacunación en tiempos de COVID-19. Revista Cubana de Pediatría, 93(3).

Carrizo, J., Betancourt, A., De Izaguirre, J., Pérez, Y., Drummond, T., Fugueroa, D., . . Ghersy, M. (2017). Esquema de inmunizaciones para niños y adolescentes de Venezuela. Recomendaciones enero 2017 Sociedad Venezolana de Puericultura y Pediatría. Archivos Venezolanos de Puericultura y Pediatría, 80(1), 34-40.

Chavez Amaro, D. M., Casanova González, M. F., Linares Pérez, N., Toledo Romaní, M. E., \& Capote Padrón, J. L. (2021). Factores de riesgo asociados a colonización nasofaríngea en niños vacunados con vacunas antineumocócicas. Cienfuegos 2015-16. Revista Habanera de Ciencias Médicas, 20(2).

Coello, C. (2021). Ecuador aplicará un esquema reducido de vacunas a los niños nacidos antes del 1 de agosto de 2021. Recuperado el 19 de octubre de 2021 de: https://www.edicionmedica.ec/secciones/salud-publica/ecuadoraplicara-un-esquema-reducido-de-vacunas-a-los-ninos-nacidos-antes-del-1-de-agosto-de-2021-98132

de las Casas, C. F. (2021). Avances en el desarrollo y el uso de las vacunas contra el SARS-CoV-2. Acta Médica Peruana, 38(1), 3-6.

Díaz, J., Cruz, L., Ferreira, E., Ferreyra, L., Delgado, G., \& García, M. (2018). Cobertura de vacunación y proporción de esquema incompleto en niños menores de siete años en México. Salud Pública de México, 60(3), 338-346. Recuperado el 23 de octubre de 2021 de:

http://www.scielo.org.mx/scielo.php?script=sci_arttext\&pid=S0036-36342018000300015

El Universo. (2021). Ministerio de Salud presenta campaña de vacunación regular a menores de 5 años. Recuperado el 1 de 12 de 2021 de: https://www.eluniverso.com/guayaquil/comunidad/ministerio-de-salud-presentacampana-de-vacunacion-regular-a-menores-de-5-anos-nota/

Escobar-Díaz, F., Osorio-Merchán, M., \& De la Hoz-Restrepo, F. (12 Abr de 2018). Motivos de no vacunación en menores de cinco años en cuatro ciudades colombianas. Revista Panamericana de Salud Píblica, 41, e123. https://doi.org/10.26633/RPSP.2017.123

Faundes, V., Pardo, R., Cammarata-Scalisi, F., Alarcon, P., Lay-Son, G., \& San Martin, E. (2021). Consenso de la Rama de Genética de la Sociedad Chilena de Pediatría sobre priorización de personas con Síndrome de Down y otras condiciones poco frecuentes en la Campaña de Vacunación COVID-19. Andes pediátrica, (AHEAD), $0-0$.

Isidro-Ríos, T. L., \& Gutiérrez-Aguado, A. (2021). Factores prenatales asociados al incumplimiento del esquema básico de vacunación en menores de 5 años. Revista de la Facultad de Medicina Humana, 21(2), 354-363.

Hernández, R., \& Mendoza, C. (2018). Metodología de la investigación. Las rutas cuantitativa, cualitativa y mixta (Tercera ed.). (Education, Ed.) Ciudad de México: Mc Graw Hill.

Martin-Fumadó, C., Aragonès, L., Areste, M. E., \& Arimany-Manso, J. (2021). Reflexiones médico-legales, éticas y deontológicas de la vacunación de COVID-19 en profesionales sanitarios. Medicina Clínica, 157, 79-84.

Mendoza-Mendoza, A., Cervantes de la Torre, K., \& De la Hoz Domínguez, E. (2020). Programas de vacunación infantil en América Latina, 2000-2015. Revista Cubana de Salud Pública, 45(3). Obtenido de Programas de vacunación infantil en América Latina, 2000-2015.

Ministerio de Salud. (2021). Inicia en octubre campaña de vacunación regular (no COVID-19) para niños y niñas menores de 5 años. Recuperado el 21 de octubre de 2021 de: https://www.salud.gob.ec/inicia-en-octubrecampana-de-vacunacion-regular-no-covid-19-para-ninos-y-ninas-menores-de-5-anos/ 
Ministerio de Salud de Argentina. (2020). Sostenimiento de la vacinación de calendario en contexto de pandemia. Recuperado el 30 de 10 de 2021 de: https://bancos.salud.gob.ar/sites/default/files/2020-09/covid19sostenimiento-de-vacunacion-de-calendario-en-contexto-de-pandemia.pdf

Ministerio de Salud Pública. (2019). Estrategia Nacional de Inmunizaciones. Recuperado el 05 de 11 de 2021 de: https://aplicaciones.msp.gob.ec/salud/archivosdigitales/documentosDirecciones/dnn/archivos/AC_00063_20 19\%20OCT\%2031.PDF

Moreta-Herrera, R., Rodas, J., \& Lara-Salazar, M. (2021). Factor validity of Alcohol use Disorders Identification Test (AUDIT) using robust estimations in Ecuadorian adolescents. Alcohol \& Alcoholism, 56(4), 482-489. https://doi.org/10.1093/alcalc/agaa126

Moyer, M. W. (2019). Reinventar las vacunas. Investigación y ciencia, 515, 66-73.

Oyola, Y., \& Murillo, E. (2020). Influencia del covid-19 en el incumplimiento del esquema de vacunación en niños menores de un año de edad (Trabajo de titulación no publicado ed.). Guayaquil: Universidad de Guayaquil. Recuperado el 30 de 10 de 2021 de Repositorio Institucional de la Universidad de Guayaquil: http://repositorio.ug.edu.ec/bitstream/redug/53891/1/1721-TESIS-OYOLA-MURILLOLIC.ALICIA\%20DONOSO.pdf

Parcon-Bitanga, M., Rodríguez-Díaz, J. L., Carrión-Morales, M. K., \& Sangoluisa-Rosales, J. M. (2019). Factores que afectan al proceso de inmunización en la comunidad étnica Chachis, Ecuador 2018. Revista Archivo Médico de Camagüey, 23(6), 709-719.

Pardo, A., Cardoso, E., Díaz, S., Díaz, L., \& Montañéz, A. (2007). Evaluación de la adherencia al esquema de vacunación Plan Ampliado de Inmunizaciones Clínica. Revista Ciencias de la Salud, 5(1), 18-32.

Porras, O. (2008). Vacunación: esquemas y recomendaciones generales. Acta pediátrica costarricense, 20, 65-76.

Sacba, V. (2020). Factores que influyen en la no adherencia al esquema de vacunación de los menores de un año, en la aldea Diraznales, Concepción Chiquirichapa, Quetzal Tenango, Guatemala (Tesis de grado no publicada ed.). Guatemala: Universidad de Guatemala.

Sangoluisa-Rosales, J., Carrión-Morales, M., Rodríguez-Díaz, J., \& Parcon-Bitanga, M. (2019). Factores que afectan al proceso de inmunización en la comunidad étnica Chachis, Ecuador 2018. Revista Archivo Médico de Camagüey, 6, 709-719.

Sarmiento, V. (2020). Factores incidentes en el cumplimiento de coberturas de vacunación en menores de 12 a 23 meses. Revista de Ciencias de la Salud más Vita, 2(1). Obtenido de https://acvenisproh.com/revistas/index.php/masvita/article/view/92

Solís, H., Lino, A., \& Plua, L. (2018). Factores socios - culturales que inciden en el cumplimiento del esquema de inmunización en niños menores de un año que acuden al Centro de Salud Puerto López (Primera ed.). (S. Calderón, Ed.) Puerto Rico: Revista Científica Dominio de las Ciencias. Obtenido de https://dialnet.unirioja.es/servlet/articulo?codigo $=6560187$

Tirado Otálvaro, A. F., \& Moreno Uribe, C. M. (2007). Factores asociados a las bajas coberturas de vacunación en Santa Bárbara (Antioquia) 2005 - 2006. Medicina UPB, 26(1), 33-42.

Trinidad, I., \& Gutiérrez, A. (2021). Factores prenatales asociados al incumplimiento del esquema básico de vacunación en menores de 5 años. Revista de la Facultad de Medicina Humana, 21(2), 354-363. Recuperado el 22 de octubre de 2021, de http://www.scielo.org.pe/scielo.php?script=sci_arttext\&pid=S2308-05312021000200354

Villalobos Vásquez, D. (2019). Factores que influyen en el cumplimiento del esquema de vacunación en niños menores de 2 años del Centro de Salud de Morales, provincia y región de San Martín, periodo octubre 2017 a febrero 2018 (Tesis no publicada ed.). Tarapoto: Universidad Nacional de San Martín. Recuperado el 30 de 10 de 2021, de Repositorio Institucional Universidad Nacional de San Martín: http://repositorio.unsm.edu.pe/bitstream/handle/11458/3226/ENFERMERIA\%20$\% 20$ Denisse $\% 20$ Villalobos $\% 20 \mathrm{~V} \%$ c3\%a1 squez.pdf?sequence=1\&isAllowed=y 